\title{
The Emergence of the GeoSharing Economy
}

\author{
Ursula Benz and Manfred Krischke
}

Over the recent years, the "sharing economy", which ranges from Airbnb to ZipCars, has emerged as a widely applicable business concept, with a substantial market potential: Various studies predict an annual volume of over 20 billion USDollars for the sharing economy (Foye 2016). The main value proposition lies in making otherwise unused resources (for example an unoccupied apartment, or a parked car) easily available to those who need them-temporarily, and on a payper-use basis. Companies like CloudEO aim to adapt this model to the market for Earth Observation services, which have lagged far behind in their potential-in the depth of geospatial information services adding value to the business workflows of the industry, as well as with respect to their overall market volume and potential.

Companies like CloudEO forecast that applying the model of the sharing economy to earth observation and geospatial solutions will trigger the long expected disruptive growth of the EO- and geomarket.

This article will discuss the basic success factors for commercial sharing solutions and the applicability for the earth observation and geomarket. Three components are essential to a successful sharing solution:

- Availability of unused or underused resources

- Prospective customers with commercial interest in these resources

- Easy access to these resources.

The ease of access itself has three main aspects: The technical, the commercial and the legal access to the resources. If any one of those three access modes is difficult or not clearly regulated, the sharing solution does not scale. This constellation clearly necessitates a platform with a platform operator who takes care of these components.

\footnotetext{
U. Benz $(\triangle) \bullet$ M. Krischke

CloudEO AG, Munich, Germany

e-mail: ubenz@cloudeo-ag.com; mkrischke@cloudeo-ag.com
} 
This can be conveniently demonstrated by looking at the concept of car sharing. Multiple successful solutions, commercially as well as community-based, have sprung up in many cities and even on a national basis. However, the same underlying concept rarely works just between two neighbours. Why is this the case? At first hand, it appears to be ridiculous that so many cars might be sitting idly in adjacent garages or carports. And since, for the average household, owning and maintaining a car consumes a significant percentage of the family income, the motivation to reduce these costs by sharing with a neighbour should be high. The physical and technical aspects of sharing - essentially just handing over the ignition key-could easily be arranged, and even the coordination of schedules and organization for neighbourto-neighbour car sharing could be worked out rather effortlessly. The challenges lie mostly in the legal and administrative complications of owning and operating a car: the complexities of insurance policies, the liabilities in case of accidents, and the often muddled responsibilities to keep the car clean and in safe driving condition quickly could become challenging just between two neighbours. What is actually missing here is a shared platform.

In all successful sharing solutions, the platform operator takes care of legal and commercial transparency, and provides the administration. Transactions between provider and user are typically arranged by a few simple rules and regulations. And the wide availability of smartphones allow the platform operators to move the transactions online, with apps that let users easily find and book resources wherever they are and whenever they need them.

Whether the platform operator enables the technical access to the resources, or this access is partially or fully provided by the owner or licensee of the resources, depends on the respective sharing solution. Examples for the first case are car and bike sharing services, where operators put large efforts in creating and facilitating easy technical access. They inspect and clean the cars, equip them with special authorization systems - and some operators actively move cars and bikes from rarely used places to locations with frequent access requests. In On the other hand, apartment-sharing solutions, for example, leave the task to provide actual access to the resources, i.e. the booked rooms, to the providers of the housings.

How does this apply to the geomarket? Is GeoSharing suited to become a successful business model?

The first requirement, the availability of unused or underused resources, is definitely met:

- Petabytes of remote sensing imagery are stored away and idling in archives, and many of them have rarely been used-if they have been used at all.

- Geosoftware and -hardware, as expensive as they are to acquire and maintain are sitting around most of the time in standby mode, waiting for the next project or mission, 
- In every value adding company, and in each of the many research projects, knowledge is developed on how to handle remote sensing data properly and how to extract reliable information from these data as these steps are mandatory pre-conditions for qualified GeoServices. But this knowledge typically is bottled up within the company, and often even within the project group; until now, there has been no viable solution to generate business with these building blocks.

But, is there a demand, a market for all these unused resources, which would be the second prerequisite, as previously stated? Most certainly. But to keep within the scope of this chapter, the following remarks will focus only the most obvious demand for archive imagery and sharing of expert knowledge.

Archived images can be a highly valuable resource to understand developments and generate reliable statistics, and quite obviously, many research and development studies could benefit from these resources. But access to this vast resource is not only valuable for its immediate informational content-many promising application developments for "big data" image processing and knowledge extraction are hampered by a lack of sufficiently large data sets for training and calibration. Which makes such applications highly situational, and the resulting analytics less transferable. When neither the real potential nor the limits of the methods are sufficiently understood, wrong decisions will be made and investments are lost. Applying these analytics to large volume of data in space and time will help to weed out less suitable approaches and further improve the viable methods-generating a quicker return of investment in the long run. It will help to transform these analytics from one-time research projects to reusable products for repeatable commercial services. This in itself will generate more people relying on the information and subsequently generating a sustainable demand for new data.

"Locked-in" expert knowledge: Currently, only a few companies are able to take advantage of the wealth of remote sensing data, since only those few companies possess the necessary GeoIT infrastructure and expertise to benefit from the full value-adding chain-from sensor data to vertical domain knowledge. And the solutions developed by (or for) these companies are often highly specific their unique needs. Developing modules that could be more widely shared by other companies in the field would require additional effort, from generalizing the tools to licensing and marketing, while generating very little revenue to offset the development cost. For that reason, such modules typically are not available in the marketplace.

But having such modules would open up the field for a large community of innovative image and mapping entrepreneurs. While it may be fairly easy to produce "nice" images from raw data, extracting meaningful information and creating consistent analytical tools and processes requires expert knowledge from scientists and researchers. This knowledge is currently "locked" in, but by creating 
a marketplace for that knowledge and therefore provide an incentive to share this knowledge - and offering specific analytical services — on a suitable platform can unlock it and drive further development of services in the field. Currently, the biggest impediment to market growth is a missing concept to make expert knowledge attractive and shareable.

\section{GeoSharing Platform Requirements}

The examples show how underused georesources impede the development of the market and waste the huge potential of GeoSharing. Thus, building a platform for GeoSharing seems to make sense-for the platform operator as well for the market.

However, current experience shows that requirements for a GeoSharing platform are complex. It needs to make accessible a very heterogeneous and technically demanding environment as well as a complex commercial and legal framework, which needs to accommodate commercial interests and satisfy regulatory and security requirements.

\section{Technical Access}

Providing technical access has become the low-hanging fruit, thanks to, the rapidly growing public and private cloud offerings that are available at steadily decreasing costs and with increasing storage and computational capacity. These hosted environments are requisite to enable access to data, software and analytics in an interactive and automated way. The task of the platform operator is to connect customers to these clouds and make them accessible with respect to the performance, security and data needs of the application.

In addition to the technical access, the platform needs to simplify access to the data. Therefore it has to connect to data catalogues and data itself. This, too, seems easily achievable: Over the course of the past few years, many data providers have already created APIs to provide access to on-line catalogues-and in some cases also directly to data.

But what is still missing is making higher-level data more easily accessible for non-experts in the field of remote sensing. The challenge is now to develop apps and analytics, as described earlier, which will unlock the expert knowledge and make it accessible for these users on an integrated GeoSharing platform.

The analytics can be assigned to four categories:

1. Transforming raw data to imagery and to calibrated pixels

2. Transfer calibrated pixels into normalized indices and bio-physical parameters 
3. Publishing tools to embed the final information into the business workflow of governmental or industrial users of geoservices

4. Generating vertical specific solutions, e.g. for agriculture or forestry

If the platform succeeds to enable solutions for categories 1, 2 and 3, it will encourage an increased usage of data. The, an increased number of category 4 solutions can be more or less taken for granted, and this will drive revenue to the GeoSharing ecosystem.

To this end, the most imminent task is to simplify the technical access. Although hosted environments make it much simpler to provide these algorithms, compared to deploying them in the very heterogeneous proprietary IT environments of the customers, the real push will only come if the providers of these analytics can easily monetize them without too much overhead-and still know that their intellectual property is safe and secure.

Unlocking the power of archived imagery will be another way how such a platform can contribute to the development of category 1 and 2 applications. Here, too, the platform needs to secure the intellectual property and the commercial interest of both the data provider and the application developer. To give an incentive to the providers who supply the developers with low-cost and easy access to data, software, and IT, this early-stage support needs to be reflected in appropriate business opportunities for the suppliers, once the services generate revenue. Possible concepts are to share the revenue of the service according to a pre-defined schema. The platform operator supports the negotiation and takes care to monitor and report usage and cash flow transparently to all stakeholders.

\section{Commercial and Legal Access}

Herein lie the most challenging tasks of a GeoSharing platform. It will be necessary to simplify the currently highly heterogeneous legal and business models, if we want to transform the current project-based value-adding market to a productized service market.

As we explained in the beginning, this will be a prerequisite to make a GeoSharing and -collaboration solution sustainable. The platform has to take eliminate the uncertainty for the user regarding the legal and contractual appropriateness of using the data, and it has to guarantee to all providers that their commercial, legal and intellectual requirements are respected and secured.

Again, cloud technology becomes the key element here. Combined with the appropriate technology, it can help to fine-tune any usage control.

This process will take time, but companies like CloudEO have already started to drive this process, and gradually, the basis for a profitable GeoSharing market will grow. The authors of this article strongly believe that multiple platforms will emerge worldwide, each with a different focus on data, or software, or specific verticals. And they will—for the benefit of the market-compete and cooperate within a healthy ecosystem (Fig. 1). 


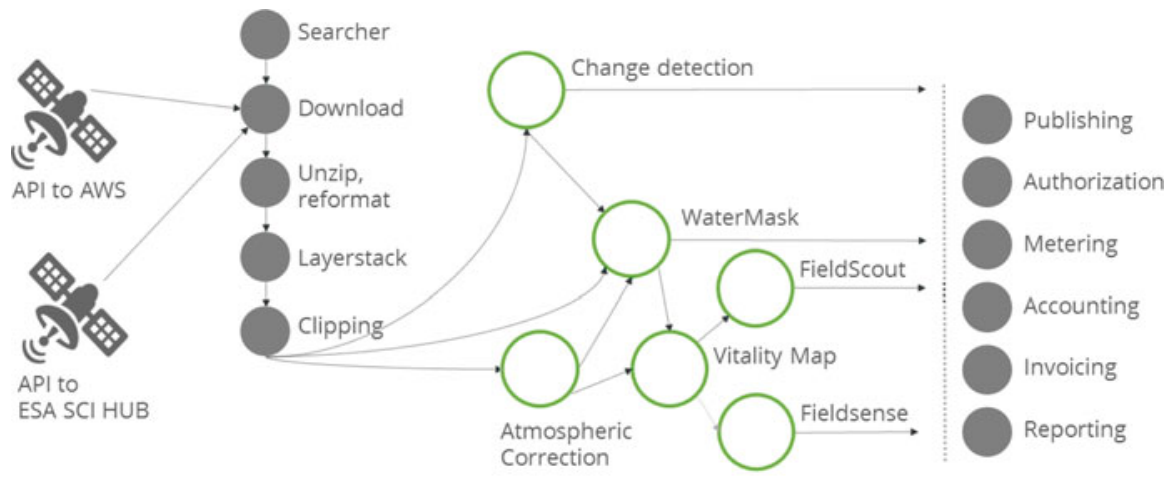

CloudEO platform services

Fig. 1 Platform services and third party analytics on CloudEO present a GeoSharing platform where the value chain is divided in many modular analytics provided by various partners. The modules can be combined as needed. Multiple applications can benefit from e.g. the analytic "Atmospheric Correction" (provider EOMAP). Whenever this analytic is used, e.g. by the analytic "WaterMask" (HySpeed Computing) or "VitalityMap" (geocledian), the provider for atmospheric correction will receive a notification and payment. The execution of the analytics and delivery of the final product to the customer is controlled by the platform. The platform provides the legal and business framework for this cooperation. Following strictly a "need to know" policy the platform ensures the full privacy of data and business intelligence of the individual partners and provides at the same time transparency and auditing. (C) CloudEO 2017, all rights reserved

\section{Reference}

Foye L (2016) Sharing economy opportunities, impacts \& disruptors 2016-2020. Jupiter, Basingstoke

Open Access This chapter is licensed under the terms of the Creative Commons Attribution 4.0 International License (http://creativecommons.org/licenses/by/4.0/), which permits use, sharing, adaptation, distribution and reproduction in any medium or format, as long as you give appropriate credit to the original author(s) and the source, provide a link to the Creative Commons license and indicate if changes were made.

The images or other third party material in this chapter are included in the chapter's Creative Commons license, unless indicated otherwise in a credit line to the material. If material is not included in the chapter's Creative Commons license and your intended use is not permitted by statutory regulation or exceeds the permitted use, you will need to obtain permission directly from the copyright holder. 